\title{
Fatal Campylobacter jejuni bacteraemia in patients with AIDS
}

\author{
ROBERTO MANFREDI, ANNA NANETTI*, MORENA FERRI* and FRANCESCO CHIODO \\ Dipartimento di Medicina Clinica Specialistica e Sperimentale, Sezione di Malattie Infettive and *Dipartimento \\ di Medicina Clinica Specialistica e Sperimentale, Sezione di Microbiologia, Università degli Studi di Bologna, \\ Via Massarenti 11, 1-40138 Bologna, Italy
}

\begin{abstract}
Two fatal cases of Campylobacter jejuni septicaemia in patients with AIDS were characterised by severe HIV-related immunodeficiency, negative stool cultures and presentation during hospitalisation, developing a clinical picture of fulminant septic shock despite therapy with appropriate antibiotics. Campylobacter spp. are important opportunist pathogens in HIV disease and may cause a septicaemic illness in the absence of enteric disease.
\end{abstract}

\section{Introduction}

Campylobacter jejuni is an important cause of acute enteritis in the general population. Infection is acquired normally through contaminated food or water, or from infected animals [1]. Extra-intestinal C. jejuni infection is rare and occurs mainly at the extreme ages of life and in immunocompromised patients, although it has been reported in otherwise healthy subjects [2]. This paper describes two HIV-infected patients with rapidly fatal C. jejuni bacteraemia.

\section{Case reports}

\section{Patient no. 1}

A 58-year-old HIV-infected patient with a previous diagnosis of AIDS due to candida oesophagitis, and suffering from other severe underlying diseases (genital herpes, disseminated cytomegalovirus infection and cachexia), developed hyper-pyrexia and signs and symptoms of bacterial septicaemia after hospitalisation for 7 days. The $\mathrm{CD}^{+}$lymphocyte count was 11 cells $/ \mu 1$, but there was no significant neutropenia. Two consecutive blood cultures yielded $C$. jejuni; the pathogen was cultured on horse-blood agar at $42^{\circ} \mathrm{C}$ in a micro-aerophilic environment, and identified by its typical microscopic appearance and specific metabolic tests. No obvious exposure to campylobacter was apparent. Gastrointestinal symptoms were absent and there was no other obvious primary focus of infection. Stool cultures were negative for Campylobacter spp.

Received 5 March 1998; revised version accepted 9 Sept. 1998.

Corresponding author: Dr R. Manfredi.
On the basis of in-vitro antimicrobial susceptibility tests, treatment was commenced with intravenous (i.v.) ciprofloxacin and co-trimoxazole. The initial clinical response was good, but the patient relapsed 4 weeks later. $C$. jejuni with the same antimicrobial susceptibility profile as the original isolate was grown from blood cultures. Stool cultures remained negative and there were no gastrointestinal features. Despite the administration of i.v. ceftriaxone $(2 \mathrm{~g} /$ day $)$ and netilmicin $(300 \mathrm{mg} /$ day), the patient developed sepsis and died 5 days later. Autopsy findings showed signs of end-organ failure due to septic shock and complicated by disseminated intravascular coagulation; CMV adrenal insufficiency was excluded.

\section{Patient no. 2}

A 28-year-old patient with full-blown AIDS, with liver cirrhosis, oesophageal candidiasis and systemic cytomegalovirus disease developed signs of septicaemia 11 days after admission to hospital. The $\mathrm{CD}^{+}{ }^{+}$count was $136 / \mu 1$ but there was no neutropenia. $C$. jejuni was isolated repeatedly from blood cultures. Apart from a history of a mild intermittent diarrhoea, gastrointestinal symptoms and signs were absent. Repeated stool cultures were negative for Campylobacter spp. Antimicrobial treatment was commenced with i.v. ceftazidime ( $3 \mathrm{~g} /$ day) and amikacin $(500 \mathrm{mg} /$ day) but the patient developed septic shock and died, despite intensive supportive care. Autopsy findings supported the clinical picture.

\section{Discussion}

Campylobacter spp. (mostly C. jejuni, but also C. fetus and $C$. coli) are important enteric pathogens in patients 
with HIV disease, who acquire this infection by either the oral or sexual route $[1,3,4]$. Campylobacter enteritis is more common in AIDS patients than in the general population [5], although invasive or disseminated infection is rarely recognised. In a 15year international literature survey (1983-1997) performed by the comprehensive Index Medicus-Medline, only 23 cases of bacteraemia caused by Campylobacter spp. were identified in HIV-infected patients, in 11 separate reports [3,5-14]. Nineteen of 23 cases were caused by $C$. jejuni $[3,5-9,13,14]$; C. fetus [10-12] and $C$. coli [10] were responsible for only three and one case, respectively. Signs and symptoms of enteric involvement or isolation of pathogens from stools, or both, were recorded in the majority of patients (16 of 22 evaluable cases) [3,6-14]. Focal complications were observed occasionally, such as cholecystitischolangitis $[6,11,12]$ and osteomyelitis [13]. Interestingly, campylobacter infection occurring during HIV disease has also been associated with immune-mediated disorders, such as thrombotic thrombocytopaenic purpura [3] and Guillain-Barré syndrome [15]. Unlike the two patients described in the present report, the outcome of HIV-related campylobacteriosis was favourable (with or without disease relapses) in 21 of 22 patients, with only one fatal case secondary to septic shock. Most episodes of campylobacter infection, including those in HIV-infected subjects, are community-acquired $[1,3,5,7]$. In both the cases described above, the campylobacter septicaemia occurred 7-11 days after admission to hospital. Severe HIV-related immunodeficiency, defined as a low $\mathrm{CD}^{+}$count, predisposes to a greater risk of septicaemia and recurrent infection $[3,5,9]$. The mean $\mathrm{CD}^{+}$count in HIV-infected patients suffering from campylobacter bacteraemia ranged from 74 to 108 cells $/ \mu 1$ in two published series $[3,8]$. Although campylobacter infections are associated usually with a low $\mathrm{CD}^{+}$count, frequency of relapse is less than with HIV-associated salmonella infections [8]. The two cases described above support the observation that bacteraemic campylobacter infections complicating HIV disease usually occur in individuals with a prior or concurrent diagnosis of AIDS $[3,5,8]$, whose survival is shorter than the mean estimated for AIDS patients [5].

More recently, campylobacter-like organisms (CLO), now included in the genus Helicobacter, have been reported as pathogens in the setting of HIV disease [5, 16-19]. Acquired by either the enteric or sexual route, $H$. cinaedi, $H$. fennelliae and $H$. laridis cause proctitis-colitis, arthritis, cellulitis and other localised infections, often complicated by persisting or relapsing septicaemia, despite antibiotic administration.

Campylobacter spp. are usually susceptible to a broad range of antimicrobial agents, including macrolides, tetracyclines, $\beta$-lactams, chloramphenicol, rifampicin and quinolones. However, treatment failures have been reported in campylobacter infections complicating
HIV disease $[3,4,9,13,14,20]$. These are generally associated with in-vitro antibiotic resistance (even against erythromycin, tetracyclines and fluoroquinolones), promoted by prolonged antimicrobial administration, and resulting in persistence of the organisms and multiple disease relapses. Helicobacter spp. isolated from HIV-infected patients have also been reported to be multi-resistant to antibiotics [17-19].

The two cases of AIDS-related $C$. jejuni bacteraemia described above were characterised by severe HIVrelated immunodeficiency, no obvious exposure to the organism and negative stool cultures, and presented after hospitalisation for $>6$ days. Both cases were fatal following the development of septic shock, despite the administration of appropriate antibiotic therapy, probably because of severe immunodeficiency and multiple underlying diseases.

In conclusion, Campylobacter spp. are important pathogens in patients with HIV infection. Septicaemia may complicate AIDS even in the absence of enteric involvement and neutropenia, and may have a severe, rapidly lethal course despite antimicrobial therapy in immunocompromised hosts. They need specific culture requirements; both Campylobacter spp. and Helicobacter spp. may fail to grow in some blood culture systems $[3,21]$, and require specialised subculture protocols. Therefore, the frequency of these infections may be underestimated, explaining the lack of reported cases of campylobacter bacteraemia in several large series of bacterial complications of HIV disease [2225].

\section{References}

1. Blaser MJ, Perez-Perez G, Smith PF et al. Extraintestinal Campylobacter jejuni and Campylobacter coli infections: host factors and strain characteristics. $J$ Infect Dis 1986; 153: 552-559.

2. Shandera WX, Tormey MP, Blaser MJ. An outbreak of bacteremic Campylobacter jejuni infection. Mt Sinai $J$ Med 1992; 59: 53-56.

3. Molina J.-M, Casin I, Hausfater $\mathrm{P}$ et al. Campylobacter infections in HIV-infected patients: clinical and bacteriological features. AIDS 1995; 9: 881-885.

4. Tee W, Mijch A, Wright E, Yung A. Emergence of multidrug resistance in Campylobacter jejuni isolates from three patients infected with human immunodeficiency virus. Clin Infect Dis 1995; 21: 634-638

5. Sorvillo FJ, Lieb LE, Waterman SH. Incidence of campylobacteriosis among patients with AIDS in Los Angeles County. $J$ Acquir Immune Defic Syndr 1991; 4: 598-602.

6. Ruiz-Contreras J, Ramos JT, Hernandez-Sampelayo $\mathrm{T}$ et al. for the Madrid HIV Pediatric Infection Collaborative Study Group. Sepsis in children with human immunodeficiency virus infection. Pediatr Infect Dis $J$ 1995; 14: 522-526.

7. Bernard E, Carles M, Pradier C, Ozouf N, Dellamonica P. Septicémies communautaires et nosocomiales chez le patient infecté par le virus de l'immunodéficience humaine. [Community-acquired and nosocomial septicemias in patients with human immunodeficiency virus infection.] Presse Med 1996; 25: $746-750$.

8. Nelson MR, Shanson DC, Hawkins DA, Gazzard BG. Salmonella, Campylobacter, and Shigella in HIV-seroposive patients. AIDS 1992; 6: 1495-1498.

9. Perlman DM, Ampel NM, Schifman RB et al. Persistent 
Campylobacter jejuni infections in patients infected with human immunodeficiency virus (HIV). Ann Intern Med 1988; 108: $540-546$

10. Bernard E, Roger PM, Carles D, Bonaldi V, Fournier JP, Dellamonica P. Diarrhea and Campylobacter infections in patients infected with the human immunodeficiency virus. J. Infect Dis 1989; 159: 143-144.

11. Costel EE, Wheeler AP, Gregg CR. Campylobacter fetus spp. fetus cholecystitis and relapsing bacteremia in a patient with acquired immunodeficiency syndrome. South Med J 1984; 77 : 927-928.

12. Wheeler AP, Gregg CR. Campylobacter bacteremia, cholyecystitis, and the acquired immunodeficiency syndrome. Ann Intern Med 1986; 105 : 804.

13. Peterson MC, Farr RW, Castiglia M. Prosthetic hip infection and bacteremia due to Campylobacter jejuni in a patient with AIDS. Clin Infect Dis 1993; 16: 439-440.

14. Pigrau C, Almirante B, Pahissa A, Bartolomé R. Campylobacter spp. bacteremia in AIDS patients. $J$ Acquir Immune Defic Syndr Hum Retrovirol 1996; 12: 93-95.

15. Mishu B, Blaser MJ. Campylobacter jejuni, human immunodeficiency virus, and the Guillain-Barré syndrome. J Infect Dis 1994; 169: 1177

16. Kemper CA, Mickelson P, Morton A, Walton B, Deresinski SC. Helicobacter (Campylobacter) fennelliae-like organisms as an important but occult cause of bacteremia in a patient with AIDS. J Infect 1993; 26: 97-101.

17. Evans TG, Riley D. Campylobacter laridis colitis in a human immunodeficiency virus-positive patient treated with a quinolone. Clin Infect Dis 1992; 15: 172-173.
18. Burman WJ, Cohn DL, Reves RR, Wilson ML. Multifocal cellulitis and monoarticular arthritis as manifestations of Helicobacter cinaedi bacteremia. Clin Infect Dis 1995; 20 564-570.

19. Kiehlbauch JA, Tauxe RV, Baker CN, Wachsmuth IK. Helicobacter cinaedi-associated bacteremia and cellulitis in immunocompromised patients. Ann Intern Med 1994; 121: 90-93.

20. Funke G, Baumann R, Penner JL, Altwegg M. Development of resistance to macrolide antibiotics in an AIDS patient treated with clarithromycin for Campylobacter jejuni diarrhea. Eur $J$ Clin Microbiol Infect Dis 1994; 13: 612-615.

21. Wang WLL, Blaser MJ. Detection of pathogenic Campylobacter species in blood culture systems $J$ Clin Microbiol 1986; 23 $709-714$.

22. Krumholz HM, Sande MA, Lo B. Community-acquired bacteremia in patients with acquired immunodeficiency syndrome: clinical presentation, bacteriology, and outcome. $A m J$ Med 1989; 86: 776-779.

23. Andiman WA, Mezger J, Shapiro E. Invasive bacterial infections in children born to women infected with human immunodeficiency virus type 1. J Pediatr 1994; 124: 846-852.

24. Meyer CN, Skinhoj P, Prag J. Bacteremia in HIV-positive and AIDS patients: incidence, species distribution, risk-factors, outcome, and influence of long-term prophylactic antibiotic treatment. Scand J Infect Dis 1994; 26: 635-642.

25. Fichtenbaum CJ, Dunagan WC, Powderly WG. Bacteremia in hospitalized patients infected with the human immunodeficiency virus: a case-control study of risk factors and outcome. $J$ Acquir Immune Defic Syndr Hum Retrovirol 1995; 8: 51-57. 